International Journal of Innovative Research in Science, Engineering and Technology

(An ISO 3297: 2007 Certified Organization)

Vol. 3, Issue 10, October 2014

\title{
Gain- Function of Two Dissimilar Warm Standby Systems Subject To Failure Due To Voltage Fluctuations and Non-Availability of Water Resulting Failure of Hydroelectric Power with Switch Failure
}

\author{
Ashok Kumar Saini \\ Associate Professor, Department of Mathematics, B.L.J.S. College, Tosham (Bhiwani) Haryana, India
}

\begin{abstract}
The energy in the flowing water can be used to produce electricity. Hydro power is one of the best, cheapest, and cleanest sources of energy. New environmental laws affected by the danger of global warming have made energy from small hydropower plants more relevant. The mathematical theory of reliability has grown out of the demands of modern technology and particularly out of experiences in World War II (1939-1945) with complex military systems although the concept of reliability is as old as man himself. In the present paper we have taken two-nonidentical warm standby system with failure time distribution as exponential and repair time distribution as general. The Role of voltage fluctuations and non-availability of water resulting failure of hydroelectric power under which the system operates plays significant role on its working. We are considering system under (i) voltage fluctuations and (ii) Non-availability of water resulting failure of hydroelectric power causing different types of failure requiring different types of repair facilities. Using semi Markov regenerative point technique we have calculated different reliability characteristics such as MTSF, reliability of the system, availability analysis in steady state, busy period analysis of the system under repair, expected number of visits by the repairman in the long run and gain-function and graphs are drawn.
\end{abstract}

KEYWORDS: warm standby, voltage fluctuations, non-availability of water resulting failure of hydroelectric power, switches failure.

\section{INTRODUCTION}

Two-unit standby system subject to environmental conditions such as shocks, change of weather conditions etc. have been discussed in reliability literature by several authors due to significant importance in defenses, industry etc.

\section{MATERIALS AND METHODS}

A random phenomenon arising through a process which developed in time in a manner controlled by probabilistic laws is called stochastic process. It is called Markovian if the future development is completely determined by the present state and is independent of the way in which state has been achieved. Mathematically, for

$\mathrm{t}_{0<} \mathrm{t}_{1<}<\mathrm{t}_{2<}$ $<\mathrm{t}_{\mathrm{n}<\mathrm{t}+1<}$

$\left.\operatorname{Pr}\left\{\mathrm{t}_{\mathrm{n}+1} \mathrm{j} \mid \mathrm{t}_{\mathrm{n}}=\mathrm{i}, \ldots \ldots \ldots \ldots \ldots, \quad \mathrm{t}_{0}=\mathrm{i}_{0}\right\}=\operatorname{Pr}_{\operatorname{Pr}\{} \mathrm{t}_{\mathrm{n}+1} \mathrm{j} \mid \mathrm{t}_{\mathrm{n}}=\mathrm{i},\right\}$

where $t_{n}$ is the state of the process at the nth transition $n=0,1,2$,

A time point at which the system history prior to the time point, is irrelevant to the system conditions is called regenerative point. A process in which each point is a regenerative point is called a regenerative process.

Let $T_{0}, T_{1}, T_{2}, \ldots \ldots \ldots \ldots \ldots \ldots . . .$. denote the epochs at which the system enters any state $\mathrm{i}$ belonging to set of regenerative states . Let $Z_{n}$ denote the state visited at epoch $T_{n}+$, i.e., just after the transition at $T_{n}$. Then $\left\{Z_{n}, T_{n}\right\}$ is a Markov renewal process. We have applied the regenerative point technique with renewal process theory 


\section{IJIRSET \\ International Journal of Innovative Research in Science, Engineering and Technology}

ISSN: 2319-8753

(An ISO 3297: 2007 Certified Organization)

Vol. 3, Issue 10, October 2014

III.ASSUMPTIONS

1.The failure time distribution is exponential whereas the repair time distribution is arbitrary of two non-identical units. 2.The repair facility is of four types :

Type I, II repair facility - When failure due to voltage fluctuations and non-availability of water resulting failure of hydroelectric power of first unit occurs respectively

Type III, IV repair facility - When failure due to voltage fluctuations and non-availability of water resulting failure of hydroelectric power of the second unit occurs respectively.

3.The repair starts immediately upon failure of units and the repair discipline is FCFS.

4.The repairs are perfect and start immediately as soon as the voltage fluctuations and non-availability of water resulting failure of hydroelectric power of the system becomes normal. The voltage fluctuations and non-availability of water in both the units do not go steep high simultaneously.

5.The failure of a unit is detected immediately and perfectly.

6.The switches are perfect and instantaneous.

7.All random variables are mutually independent.

\section{SYMBOLS FOR STATES OF THE SYSTEM}

\section{Superscripts O, WS, SO, FVF, FNAW, SFO}

Operative, Warm Standby, Stops the operation, failure due to voltage fluctuations, failure due to non-availability of water resulting failure of hydroelectric power, Switch failed but operable respectively

Subscripts nvf, vf, nawf, ur, wr, uR

No voltage fluctuations, voltage fluctuations, non-availability of water resulting failure of hydroelectric power, under repair, waiting for repair, under repair continued respectively

Up states $-0,1,2,9 ; \quad$ Down states $-3,4,5,6,7,8,10,11 ; \quad$ Regeneration point $-0,1,2,4,7,10$

\section{V.STATES OF THE SYSTEM}

$\mathbf{0}\left(\mathbf{O}_{\text {nvf }}, \mathbf{W S}_{\text {nvf }}\right)$ One unit is operative and the other unit is warm standby and there are no voltage fluctuations and no non-availability of water resulting failure of hydroelectric power in both the units.

$\mathbf{1}\left(\mathbf{S O}_{\mathrm{nv}}, \mathbf{O}_{\mathrm{nvf}}\right)$ The operation of the first unit stops automatically due to voltage fluctuations and warm standby unit's starts operating with no voltage fluctuations.

$\mathbf{2}\left(\mathbf{F V F}_{\mathrm{ur}}, \mathbf{O}_{\mathrm{nvf}}\right)$ The first unit fails and undergoes repair after the voltage fluctuations are over and the other unit continues to be operative with no voltage fluctuations.

$\mathbf{3}\left(\mathbf{F V F}_{\mathrm{uR}}, \mathbf{S O}_{\text {uvf }}\right)$ The repair of the first unit is continued from state 2 and the operation of second unit stops automatically due to voltage fluctuations.

$\mathbf{4}\left(\mathbf{F V F}_{\text {ur }}, \mathbf{S O}_{\text {nawf }}\right)$ The first unit fails and undergoes repair after the voltage fluctuations are over and the other unit also stops automatically due to non-availability of water resulting failure of hydroelectric power.

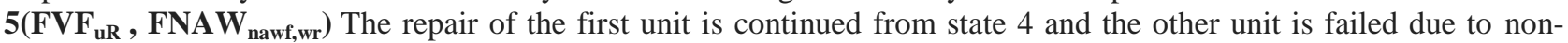
availability of water resulting failure of hydroelectric power in it \& is waiting for repair.

$\mathbf{6}\left(\mathbf{O}_{\text {nvf, }} \mathbf{F N A W _ { u r }}\right)$ the first unit becomes operative with no voltage fluctuations and the second unit is failed due to high non-availability of water is under repair.

$\mathbf{7}\left(\mathbf{S O}_{\text {nawf }}, \mathbf{S F O} \mathbf{O}_{\text {nvf, ur }}\right)$ The operation of the first unit stops automatically due to non-availability of water resulting failure of hydroelectric power and during switchover to the second unit switch fails and undergoes repair and there is no voltage fluctuations.

$\mathbf{8}\left(\mathbf{F N A W}_{\text {nawf, wr }}, \mathbf{S F O}_{\text {nvf, uR }}\right)$ The repair of failed switch is continued from state 7 and the first unit is failed after nonavailability of water resulting failure of hydroelectric power is waiting for repair.

$\mathbf{9}\left(\mathrm{O}_{\text {nvf }}, \mathbf{S O}_{\text {nawf }}\right)$ The first unit is operative with no voltage fluctuations and the operation of warm standby second unit is stopped automatically due to non-availability of water resulting failure of hydroelectric power. 


\section{(3) \\ IJIRSET \\ International Journal of Innovative Research in Science, Engineering and Technology \\ (An ISO 3297: 2007 Certified Organization)}

ISSN: 2319-8753

Vol. 3, Issue 10, October 2014

$\mathbf{1 0}\left(\mathbf{S O}_{\text {nawf }}, \mathbf{S F}_{\text {ur }}\right)$ The operation of the first unit stops automatically due to non-availability of water resulting failure of hydroelectric power and the second unit switch fails and undergoes repair after the non-availability of water resulting failure of hydroelectric power is over.

11(FNAW nawf, wr $_{\text {, }} \mathbf{S F}_{\mathbf{u R}}$ ) The repair of the second unit is continued from state 10 and the first unit is failed due to nonavailability of water resulting failure of hydroelectric power is waiting for repair.

\section{VI.TRANSITION PROBABILITIES}

Simple probabilistic considerations yield the following expressions :

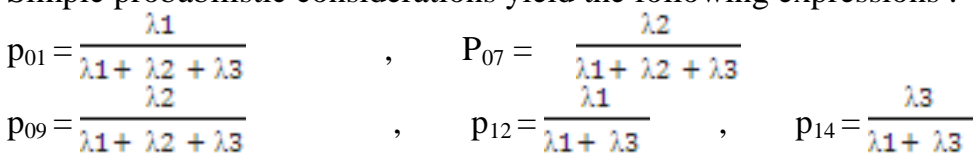

where

$\mathrm{P}_{20}=\mathrm{G}_{1}{ }^{*}\left(\lambda_{1}\right), \mathrm{P}_{22}{ }^{(3)}=\mathrm{G}_{1}{ }^{*}\left(\lambda_{1}\right)=\mathrm{p}_{23} \quad, \mathrm{P}_{72}=\mathrm{G}_{2}{ }^{*}\left(\lambda_{4}\right), \mathrm{P}_{72}{ }^{(8)}=\mathrm{G}_{2}{ }^{*}\left(\lambda_{4}\right)=\mathrm{P}_{78}$

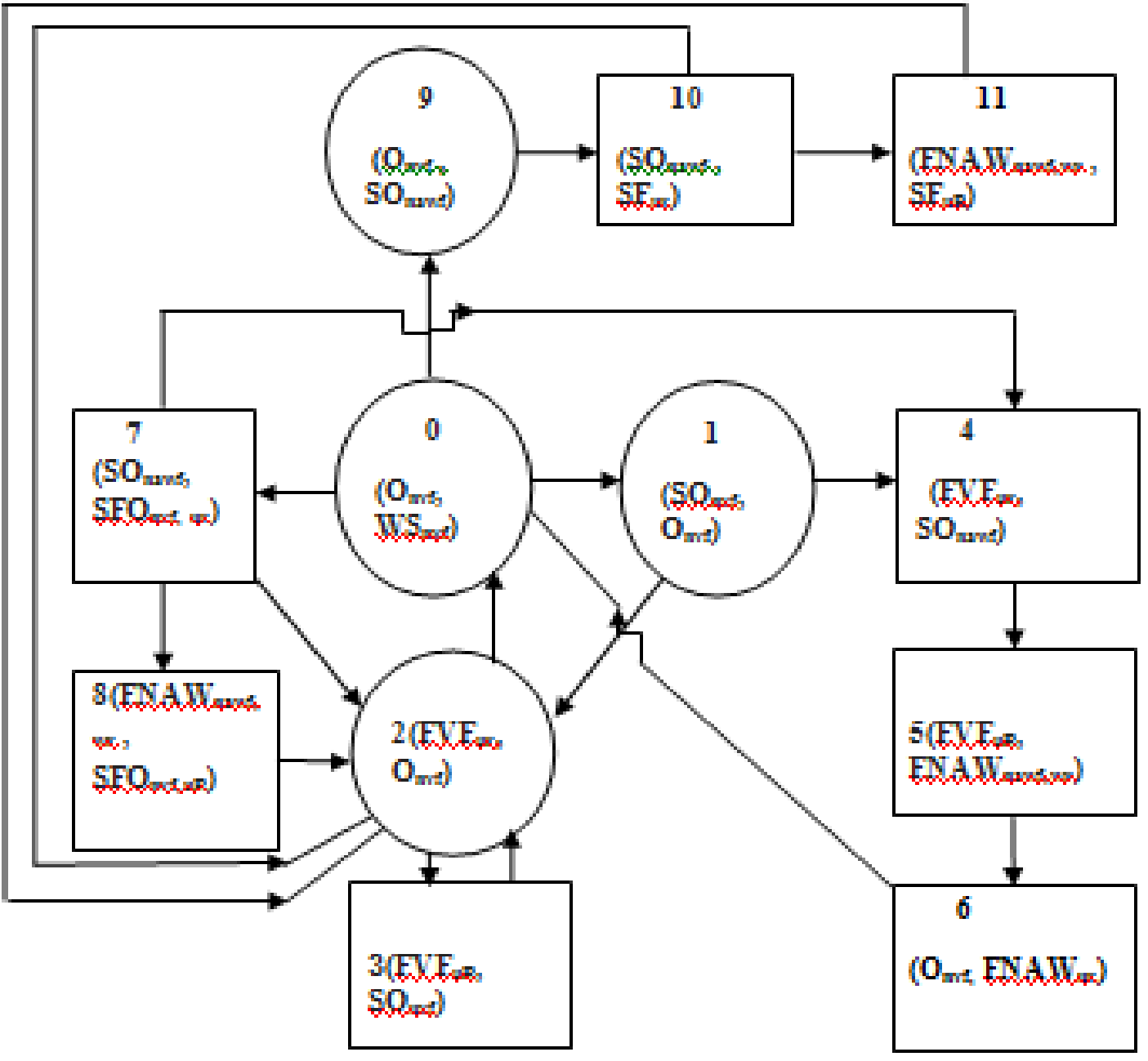

Fuli Tho State Trangitan Dhagran

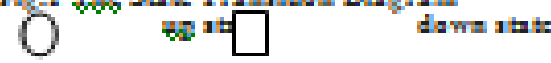




\section{IJIRSET \\ International Journal of Innovative Research in Science, Engineering and Technology}

ISSN: 2319-8753

(An ISO 3297: 2007 Certified Organization)

Vol. 3, Issue 10, October 2014

We can easily verify that

$\mathrm{p}_{01}+\mathrm{p}_{07}+\mathrm{p}_{09}=1, \mathrm{p}_{12}+\mathrm{p}_{14}=1, \mathrm{p}_{20}+\mathrm{p}_{23}\left(=\mathrm{p}_{22}{ }^{(3)}\right)=1, \mathrm{p}_{46}{ }^{(6)}=1 \mathrm{p}_{60}=1$,

$\mathrm{p}_{72}+\mathrm{P}_{72}{ }^{(5)}+\mathrm{p}_{74}=1, \mathrm{p}_{9,10}=1, \quad \mathrm{p}_{10,2}+\mathrm{p}_{10,2}{ }^{(11)}=1$

And mean sojourn time is

$\mu_{0}=\mathrm{E}(\mathrm{T})=\int_{0}^{m} P[T>t] d t$

\section{MEAN TIME TO SYSTEM FAILURE}

We can regard the failed state as absorbing

$$
\begin{aligned}
& \theta_{0}(t)=Q_{01}(t)[s] \theta_{1}(t)+Q_{09}(t)[s] \theta_{9}(t)+Q_{02}(t) \\
& \theta_{1}(t)=Q_{12}(t)[s] \theta_{2}(t)+Q_{14}(t), \theta_{2}(t)=Q_{20}(t)[s] \theta_{0}(t)+Q_{22}^{[3]}(t) \\
& \theta_{4}(t)=Q_{9,10}(t)
\end{aligned}
$$

Taking Laplace-Stiltjes transform of eq. (3-5) and solving for

$$
Q_{0}^{8}(s)=\mathrm{N}_{1}(\mathrm{~s}) / \mathrm{D}_{1}(\mathrm{~s})
$$

where

$$
\begin{aligned}
& \mathrm{N}_{1}(\mathrm{~s})=Q_{01}^{8}(s) \quad\left\{\quad Q_{12}^{8}(s) Q_{22}^{(3) 8}(s)+Q_{14}^{8}(s)\right\}+Q_{09}^{8}(s) Q_{9,10}^{8}(s)+Q_{07}^{8}(s) \\
& \mathrm{D}_{1}(\mathrm{~s})=1-Q_{01}^{8}(s) \quad Q_{12}^{8}(s) Q_{20}^{8}(s)
\end{aligned}
$$

Making use of relations (1) \& (2) it can be shown that $\theta_{0}{ }^{*}(0)=1$, which implies that $\theta_{0}(\mathrm{t})$ is a proper distribution.

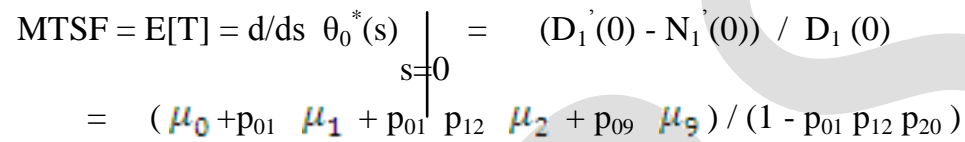

where

$\mu_{0}=\mu_{01}+\mu_{07}+\mu_{09}, \quad \mu_{1}=\mu_{12}+\mu_{14}, \mu_{2}=\mu_{20}+\mu_{22}^{(3)}, \mu_{9}=\mu_{9,10}$

\section{VIII.AVAILABILITY ANALYSIS}

Let $\mathrm{M}_{\mathrm{i}}(\mathrm{t})$ be the probability of the system having started from state I is up at time $\mathrm{t}$ without making any other regenerative state. By probabilistic arguments, we have

The value of $M_{0}(t), M_{1}(t), M_{2}(t), M_{4}(t)$ can be found easily.

The point wise availability $\mathrm{A}_{\mathrm{i}}(\mathrm{t})$ have the following recursive relations

$\mathrm{A}_{0}(\mathrm{t})=\mathrm{M}_{0}(\mathrm{t})+\mathrm{q}_{01}(\mathrm{t})[\mathrm{c}] \mathrm{A}_{1}(\mathrm{t})+\mathrm{q}_{07}(\mathrm{t})[\mathrm{c}] \mathrm{A}_{7}(\mathrm{t})+\mathrm{q}_{09}(\mathrm{t})[\mathrm{c}] \mathrm{A}_{9}(\mathrm{t})$

$\mathrm{A}_{1}(\mathrm{t})=\mathrm{M}_{1}(\mathrm{t})+\mathrm{q}_{12}(\mathrm{t})[\mathrm{c}] \mathrm{A}_{2}(\mathrm{t})+\mathrm{q}_{14}(\mathrm{t})[\mathrm{c}] \mathrm{A}_{4}(\mathrm{t}), \mathrm{A}_{2}(\mathrm{t})=\mathrm{M}_{2}(\mathrm{t})+\mathrm{q}_{20}(\mathrm{t})[\mathrm{c}] \mathrm{A}_{0}(\mathrm{t})+\mathrm{q}_{22}{ }^{(3)}(\mathrm{t})[\mathrm{c}] \mathrm{A}_{2}(\mathrm{t})$

$\mathrm{A}_{4}(\mathrm{t})=\mathrm{q}_{46}{ }^{(3)}(\mathrm{t})[\mathrm{c}] \mathrm{A}_{6}(\mathrm{t}), \mathrm{A}_{6}(\mathrm{t})=\mathrm{q}_{60}(\mathrm{t})[\mathrm{c}] \mathrm{A}_{0}(\mathrm{t})$

$\mathrm{A}_{7}(\mathrm{t})=\left(\mathrm{q}_{72}(\mathrm{t})+\mathrm{q}_{72}{ }^{(8)}(\mathrm{t})\right)[\mathrm{c}] \mathrm{A}_{2}(\mathrm{t})+\mathrm{q}_{74}(\mathrm{t})[\mathrm{c}] \mathrm{A}_{4}(\mathrm{t})$

$\mathrm{A}_{9}(\mathrm{t})=\mathrm{M}_{9}(\mathrm{t})+\mathrm{q}_{9,10}(\mathrm{t})[\mathrm{c}] \mathrm{A}_{10}(\mathrm{t}), \quad \mathrm{A}_{10}(\mathrm{t})=\mathrm{q}_{10,2}(\mathrm{t})[\mathrm{c}] \mathrm{A}_{2}(\mathrm{t})+\mathrm{q}_{10,2}{ }^{(11)}(\mathrm{t})[\mathrm{c}] \mathrm{A}_{2}(\mathrm{t})$

(7-14)

Taking Laplace Transform of eq. (7-14) and solving for $\hat{A}_{0}(s)$

where

$$
\widehat{A}_{0}(s)=\mathrm{N}_{2}(\mathrm{~s}) / \mathrm{D}_{2}(\mathrm{~s})
$$

$\mathrm{N}_{2}(\mathrm{~s})=\left(1-\hat{q}_{22}{ }^{(3)}(\mathrm{s})\right)\left\{\widehat{M}_{0}(\mathrm{~s})+\hat{q}_{01}(\mathrm{~s}) \widehat{M}_{1}(\mathrm{~s})+\hat{q}_{09}(\mathrm{~s}) \widehat{M}_{9}(\mathrm{~s})\right\}+\widehat{M}_{2}(\mathrm{~s})\left\{\hat{q}_{01}(\mathrm{~s}) \hat{q}_{42}(\mathrm{~s})+\widehat{q}_{07}(\mathrm{~s})\left(\hat{q}_{72}(\mathrm{~s})+\right.\right.$ $\left.\left.\hat{q}_{73}{ }^{(8)}(\mathrm{s})\right)+\hat{q}_{09}(\mathrm{~s}) \hat{q}_{9,10}(\mathrm{~s})\left(\hat{q}_{10,2}(\mathrm{~s})+\hat{q}_{10,2}{ }^{(11)}(\mathrm{s})\right)\right\}$

$\mathrm{D}_{2}(\mathrm{~s})=\left(1-\hat{q}_{22}{ }^{(3)}(\mathrm{s})\right)\left\{1-\hat{q}_{46}{ }^{(5)}(\mathrm{s}) \hat{q}_{60}(\mathrm{~s})\left(\hat{q}_{01}(\mathrm{~s}) \hat{q}_{44}(\mathrm{~s})+\hat{q}_{07}(\mathrm{~s}) \hat{q}_{74}(\mathrm{~s})\right)\right.$

$$
-\hat{q}_{20}(\mathrm{~s})\left\{\hat{q}_{01}(\mathrm{~s}) \hat{q}_{12}(\mathrm{~s})+\hat{q}_{07}(\mathrm{~s})\left(\hat{q}_{72}(\mathrm{~s})\right)+\hat{q}_{72}{ }^{(8)}(\mathrm{s})+\hat{q}_{09}(\mathrm{~s}) \hat{q}_{9,10}(\mathrm{~s})\left(\hat{q}_{10,2}(\mathrm{~s})+\hat{q}_{10,2}{ }^{(11)}(\mathrm{s})\right)\right\}
$$

The steady state availability 


\section{International Journal of Innovative Research in Science, Engineering and Technology}

(An ISO 3297: 2007 Certified Organization)

Vol. 3, Issue 10, October 2014

$$
\mathrm{A}_{0}=\lim _{t \rightarrow \infty x}\left[A_{0}(t)\right]=\lim _{s \rightarrow 0}\left[s \hat{A}_{0}(s)\right]=\lim _{s \rightarrow 0} \frac{s N_{2}(s)}{D_{2}(s)}
$$

Using L' Hospitals rule, we get

$$
\mathrm{A}_{0}=\lim _{s \rightarrow 0} \frac{N_{2}(s)+s N_{2}(s)}{D_{2}(s)}=\frac{N_{2}(0)}{D_{2}(0)}
$$

Where

$$
\begin{aligned}
& \mathrm{N}_{2}(0)=\mathrm{p}_{20}\left(\widehat{M}_{0}(0)+\mathrm{p}_{01} \widehat{M}_{1}(0)+\mathrm{p}_{09} \widehat{M}_{9}(0)\right)+\widehat{M}_{2}(0)\left(\mathrm{p}_{01} \mathrm{p}_{12}+\mathrm{p}_{07}\left(\mathrm{p}_{72}+\mathrm{p}_{72}^{(8)}+\mathrm{p}_{09}\right)\right) \\
& \mathrm{D}_{2}^{\prime}(0)=\mathrm{p}_{20}\left\{\mu_{0}+\mathrm{p}_{01} \mu_{1}+\left(\mathrm{p}_{01} \mathrm{p}_{14}+\mathrm{p}_{07} \mathrm{p}_{74}\right) \mu_{4}+\mathrm{p}_{07} \mu_{7}+\mathrm{p}_{07} \mu_{7}+\mathrm{p}_{09}\left(\mu_{9}+\mu_{10}\right)+\right. \\
& \quad \mu_{2}\left\{1-\left(\left(\mathrm{p}_{01} \mathrm{p}_{14}+\mathrm{p}_{07} \mathrm{p}_{74}\right)\right\}\right. \\
& \mu_{4}=\mu \frac{(5)}{{ }_{46}}, \mu_{7}=\mu_{72}+\mu \frac{(8)}{72}+\mu_{74}, \quad \mu_{10}=\mu_{10,2}+\mu \frac{(11)}{102}
\end{aligned}
$$

The expected up time of the system in $(0, t]$ is

$$
\lambda_{\mathrm{u}}(\mathrm{t})=\int_{0}^{\infty} A_{0}(\mathrm{z}) d z \quad \text { So that } \overline{\lambda_{\mathrm{u}}}(\mathrm{s})=\frac{\widehat{\mathrm{A}_{0}}(\mathrm{~s})}{\mathrm{a}}=\frac{N_{\mathrm{z}}(S)}{S D_{\mathrm{z}}(S)}
$$

The expected down time of the system in $(0, t]$ is

$$
\lambda_{\mathrm{d}}(\mathrm{t})=\mathrm{t}-\lambda_{\mathrm{u}}(\mathrm{t}) \quad \text { So that } \widehat{\lambda_{\mathrm{d}}}(\mathrm{s})=\frac{1}{\mathrm{~s}^{\mathrm{x}}}-\overrightarrow{\lambda_{\mathrm{u}}}(\mathrm{s})
$$

\section{IX.THE EXPECTED BUSY PERIOD OF THE SERVER FOR REPAIRING THE FAILED UNIT UNDER} NON-AVAILABILITY OF WATER RESULTING FAILURE OF HYDROELECTRIC POWER IN $(0$, T]

$$
\begin{aligned}
\mathrm{R}_{0}(\mathrm{t}) & =\mathrm{S}_{0}(\mathrm{t})+\mathrm{q}_{01}(\mathrm{t})[\mathrm{c}] \mathrm{R}_{1}(\mathrm{t})+\mathrm{q}_{07}(\mathrm{t})[\mathrm{c}] \mathrm{R}_{7}(\mathrm{t})+\mathrm{q}_{09}(\mathrm{t})[\mathrm{c}] \mathrm{R}_{9}(\mathrm{t}) \\
\mathrm{R}_{1}(\mathrm{t}) & =\mathrm{S}_{1}(\mathrm{t})+\mathrm{q}_{12}(\mathrm{t})[\mathrm{c}] \mathrm{R}_{2}(\mathrm{t})+\mathrm{q}_{14}(\mathrm{t})[\mathrm{c}] \mathrm{R}_{4}(\mathrm{t}) \\
\mathrm{R}_{2}(\mathrm{t}) & =\mathrm{q}_{20}(\mathrm{t})[\mathrm{c}] \mathrm{R}_{0}(\mathrm{t})+\mathrm{q}_{22}\left({ }^{3}(\mathrm{t})[\mathrm{c}] \mathrm{R}_{2}(\mathrm{t})\right. \\
\mathrm{R}_{4}(\mathrm{t}) & =\mathrm{q}_{46}\left({ }^{3}\right)(\mathrm{t})[\mathrm{c}] \mathrm{R}_{6}(\mathrm{t}) \quad, \quad \mathrm{R}_{6}(\mathrm{t})=\mathrm{q}_{60}(\mathrm{t})[\mathrm{c}] \mathrm{R}_{0}(\mathrm{t}) \\
\mathrm{R}_{7}(\mathrm{t}) & =\left(\mathrm{q}_{72}(\mathrm{t})+\mathrm{q}_{72}(8)(\mathrm{t})\right)[\mathrm{c}] \mathrm{R}_{2}(\mathrm{t})+\mathrm{q}_{74}(\mathrm{t})[\mathrm{c}] \mathrm{R}_{4}(\mathrm{t}) \\
\mathrm{R}_{9}(\mathrm{t}) & =\mathrm{S}_{9}(\mathrm{t})+\mathrm{q}_{9,10}(\mathrm{t})[\mathrm{c}] \mathrm{R}_{10}(\mathrm{t}), \quad \mathrm{R}_{10}(\mathrm{t})=\mathrm{q}_{10,2}(\mathrm{t})+\mathrm{q}_{10,2}^{(11)}(\mathrm{t})[\mathrm{c}] \mathrm{R}_{2}(\mathrm{t})
\end{aligned}
$$

Taking Laplace Transform of eq. (19-26) and solving for $\widehat{R_{0}}(s)$

$$
\widehat{R_{0}}(s)=\mathrm{N}_{3}(\mathrm{~s}) / \mathrm{D}_{2}(\mathrm{~s})
$$

where

$\mathrm{N}_{2}(\mathrm{~s})=\left(1-\hat{q}_{22}{ }^{(3)}(\mathrm{s})\right)\left\{\hat{S}_{0}(\mathrm{~s})+\hat{q}_{01}(\mathrm{~s}) \hat{S}_{1}(\mathrm{~s})+\hat{q}_{09}(\mathrm{~s}) \hat{S}_{9}(\mathrm{~s})\right\}$ and $\mathrm{D}_{2}(\mathrm{~s})$ is already defined.

$$
\text { In the long run, } \quad \mathrm{R}_{0}=\frac{\mathrm{N}_{\mathrm{g}}(0)}{D_{\mathrm{z}}(0)}
$$

where $\mathrm{N}_{3}(0)=\mathrm{p}_{20}\left(\hat{S}_{0}(0)+\mathrm{p}_{01} \hat{S}_{1}(0)+\mathrm{p}_{09} \hat{S}_{9}(0)\right)$ and $\mathrm{D}_{2}^{\prime}(0)$ is already defined.

The expected period of the system under non-availability of water resulting failure of hydroelectric power in $(0, \mathrm{t}]$ is $\lambda_{r v}(\mathrm{t})=\int_{0}^{\infty} R_{0}(z) d z$ So that $\widehat{\lambda_{r v}}(\mathrm{~s})=\frac{\widehat{\mathbb{R}}_{Q}(\mathrm{~s})}{\mathrm{a}}$

\section{X.THE EXPECTED BUSY PERIOD OF THE SERVER FOR REPAIRING THE FAILED UNITS UNDER VOLTAGE FLUCTUATIONS BY THE REPAIRMAN IN $(0, T]$}

$\mathrm{B}_{0}(\mathrm{t})=\mathrm{q}_{01}(\mathrm{t})[\mathrm{c}] \mathrm{B}_{1}(\mathrm{t})+\mathrm{q}_{07}(\mathrm{t})[\mathrm{c}] \mathrm{B}_{7}(\mathrm{t})+\mathrm{q}_{09}(\mathrm{t})[\mathrm{c}] \mathrm{B}_{9}(\mathrm{t})$

$\mathrm{B}_{1}(\mathrm{t})=\mathrm{q}_{12}(\mathrm{t})[\mathrm{c}] \mathrm{B}_{2}(\mathrm{t})+\mathrm{q}_{14}(\mathrm{t})[\mathrm{c}] \mathrm{B}_{4}(\mathrm{t}), \mathrm{B}_{2}(\mathrm{t})=\mathrm{q}_{20}(\mathrm{t})[\mathrm{c}] \mathrm{B}_{0}(\mathrm{t})+\mathrm{q}_{22}{ }^{(3)}(\mathrm{t})[\mathrm{c}] \mathrm{B}_{2}(\mathrm{t})$

$\mathrm{B}_{4}(\mathrm{t})=\mathrm{T}_{4}(\mathrm{t})+\mathrm{q}_{46}{ }^{(3)}(\mathrm{t})[\mathrm{c}] \mathrm{B}_{6}(\mathrm{t}), \quad \mathrm{B}_{6}(\mathrm{t})=\mathrm{T}_{6}(\mathrm{t})+\mathrm{q}_{60}(\mathrm{t})[\mathrm{c}] \mathrm{B}_{0}(\mathrm{t})$

$\mathrm{B}_{7}(\mathrm{t})=\left(\mathrm{q}_{72}(\mathrm{t})+\mathrm{q}_{72}{ }^{(8)}(\mathrm{t})\right)[\mathrm{c}] \mathrm{B}_{2}(\mathrm{t})+\mathrm{q}_{74}(\mathrm{t})[\mathrm{c}] \mathrm{B}_{4}(\mathrm{t})$

$\mathrm{B}_{9}(\mathrm{t})=\mathrm{q}_{9,10}(\mathrm{t})[\mathrm{c}] \mathrm{B}_{10}(\mathrm{t}), \mathrm{B}_{10}(\mathrm{t})=\mathrm{T}_{10}(\mathrm{t})+\left(\mathrm{q}_{10,2}(\mathrm{t})+\mathrm{q}_{10,2}{ }^{(11)}(\mathrm{t})[\mathrm{c}] \mathrm{B}_{2}(\mathrm{t})\right.$

Taking Laplace Transform of eq. (29-36) and solving for $\widehat{B_{0}}(s)$ 


\section{International Journal of Innovative Research in Science, Engineering and Technology}

(An ISO 3297: 2007 Certified Organization)

Vol. 3, Issue 10, October 2014

$$
\widetilde{B_{0}}(s) \quad=\mathrm{N}_{4}(\mathrm{~s}) / \mathrm{D}_{2}(\mathrm{~s})
$$

where

$\mathrm{N}_{4}(\mathrm{~s})=\left(1-\hat{q}_{22}{ }^{(3)}(\mathrm{s})\right)\left\{\hat{q}_{01}(\mathrm{~s}) \hat{q}_{14}(\mathrm{~s})\left(\widehat{T}_{4}(\mathrm{~s})+\hat{q}_{46}{ }^{(5)}(\mathrm{s}) \widehat{T}_{6}(\mathrm{~s})\right)+\widehat{q}_{07}{ }^{(3)}(\mathrm{s}) \quad \widehat{q}_{74}(\mathrm{~s})\left(\widehat{T}_{4}(\mathrm{~s})\right.\right.$

$$
\left.\left.+\hat{q}_{46}{ }^{(5)}(\mathrm{s}) \widehat{T}_{6}(\mathrm{~s})\right)+\hat{q}_{09}(\mathrm{~s}) \widehat{q}_{09,10}(\mathrm{~s}) \widehat{T}_{10}(\mathrm{~s})\right)
$$

And $\mathrm{D}_{2}(\mathrm{~s})$ is already defined.

In steady state, $\mathrm{B}_{0}=\frac{N_{4}(0)}{D_{2}(0)}$

where $\mathrm{N}_{4}(0)=\mathrm{p}_{20}\left\{\left(\mathrm{p}_{01} \mathrm{p}_{14}+\mathrm{p}_{07} \mathrm{p}_{74}\right)\left(\widehat{T}_{4}(0)+\widehat{T}_{6}(0)\right)+\mathrm{p}_{09} \widehat{T}_{10}(0)\right\}$ and $\mathrm{D}_{2}^{\prime}(0)$ is already defined.

The expected busy period of the server for repair in $(0, t]$ is

$\lambda_{r u}(\mathrm{t})=\int_{0}^{\infty} B_{0}(z) d z \quad$ So that $\widehat{\lambda_{r u}}(\mathrm{~s})=\frac{\widehat{\mathrm{B}}_{0}(\mathrm{~s})}{\mathrm{s}}$

\section{THE EXPECTED BUSY PERIOD OF THE SERVER FOR REPAIR OF SWITCH IN $(0, T]$}

$\mathrm{P}_{0}(\mathrm{t})=\mathrm{q}_{01}(\mathrm{t})[\mathrm{c}] \mathrm{P}_{1}(\mathrm{t})+\mathrm{q}_{07}(\mathrm{t})[\mathrm{c}] \mathrm{P}_{7}(\mathrm{t})+\mathrm{q}_{09}(\mathrm{t})[\mathrm{c}] \mathrm{P}_{9}(\mathrm{t})$

$\mathrm{P}_{1}(\mathrm{t})=\mathrm{q}_{12}(\mathrm{t})[\mathrm{c}] \mathrm{P}_{2}(\mathrm{t})+\mathrm{q}_{14}(\mathrm{t})[\mathrm{c}] \mathrm{P}_{4}(\mathrm{t}), \mathrm{P}_{2}(\mathrm{t})=\mathrm{q}_{20}(\mathrm{t})[\mathrm{c}] \mathrm{P}_{0}(\mathrm{t})+\mathrm{q}_{22}{ }^{(3)}(\mathrm{t})[\mathrm{c}] \mathrm{P}_{2}(\mathrm{t})$

$\mathrm{P}_{4}(\mathrm{t})=\mathrm{q}_{46}(\mathrm{~s})(\mathrm{t})[\mathrm{c}] \mathrm{P}_{6}(\mathrm{t}), \quad \mathrm{P}_{6}(\mathrm{t})=\mathrm{q}_{60}(\mathrm{t})[\mathrm{c}] \mathrm{P}_{0}(\mathrm{t})$

$\mathrm{P}_{7}(\mathrm{t})=\mathrm{L}_{7}(\mathrm{t})+\left(\mathrm{q}_{72}(\mathrm{t})+\mathrm{q}_{72}{ }^{(8)}(\mathrm{t})\right)[\mathrm{c}] \mathrm{P}_{2}(\mathrm{t})+\mathrm{q}_{74}(\mathrm{t})[\mathrm{c}] \mathrm{P}_{4}(\mathrm{t})$

$\mathrm{P}_{9}(\mathrm{t})=\mathrm{q}_{9,10}(\mathrm{t})[\mathrm{c}] \mathrm{P}_{10}(\mathrm{t}), \quad \mathrm{P}_{10}(\mathrm{t})=\left(\mathrm{q}_{10,2}(\mathrm{t})+\mathrm{q}_{10,2}{ }^{(11)}(\mathrm{t})\right)[\mathrm{c}] \mathrm{P}_{2}(\mathrm{t}) \quad(40-47)$

Taking Laplace Transform of eq. (40-47) and solving for

$$
\widetilde{P}_{0}^{-}(s)=\mathrm{N}_{5}(\mathrm{~s}) / \mathrm{D}_{2}(\mathrm{~s})
$$

where $\mathrm{N}_{2}(\mathrm{~s})=\widehat{q}_{07}(\mathrm{~s}) \widehat{L}_{7}(\mathrm{~s})\left(1-\hat{q}_{22}{ }^{(3)}(\mathrm{s})\right)$ and $\mathrm{D}_{2}(\mathrm{~s})$ is defined earlier.

In the long run, $\mathrm{P}_{0}=\frac{N_{s}(0)}{D_{2}(0)}$

where

$\mathrm{N}_{5}(0)=\mathrm{p}_{20} \mathrm{p}_{07} \widehat{L}_{4}(0)$ and $\mathrm{D}_{2}^{\prime}(0)$ is already defined.

The expected busy period of the server for repair of the switch in $(0, t]$ is

$\lambda_{r g}(\mathrm{t})=\int_{0}^{\infty} P_{0}(z) d z \quad$ So that $\widehat{\lambda}_{r s}(\mathrm{~s})=\frac{\hat{\mathrm{P}}_{\mathrm{Q}}(\mathrm{q})}{\mathrm{a}}$

\section{THE EXPECTED NUMBER OF VISITS BY THE REPAIRMAN FOR REPAIRING THE DISSIMILAR UNITS IN $(0, T]$}

$\mathrm{H}_{0}(\mathrm{t})=\mathrm{Q}_{01}(\mathrm{t})[\mathrm{c}] \mathrm{H}_{1}(\mathrm{t})+\mathrm{Q}_{07}(\mathrm{t})[\mathrm{c}] \mathrm{H}_{7}(\mathrm{t})+\mathrm{Q}_{09}(\mathrm{t})[\mathrm{c}] \mathrm{H}_{9}(\mathrm{t})$

$\mathrm{H}_{1}(\mathrm{t})=\mathrm{Q}_{12}(\mathrm{t})[\mathrm{c}]\left[1+\mathrm{H}_{2}(\mathrm{t})\right]+\mathrm{Q}_{14}(\mathrm{t})[\mathrm{c}]\left[1+\mathrm{H}_{4}(\mathrm{t})\right], \mathrm{H}_{2}(\mathrm{t})=\mathrm{Q}_{20}(\mathrm{t})[\mathrm{c}] \mathrm{H}_{0}(\mathrm{t})+\mathrm{Q}_{22}{ }^{(3)}(\mathrm{t})[\mathrm{c}] \mathrm{H}_{2}(\mathrm{t})$

$\mathrm{H}_{4}(\mathrm{t})=\mathrm{Q}_{46}{ }^{(3)}(\mathrm{t})[\mathrm{c}] \mathrm{H}_{6}(\mathrm{t}), \mathrm{H}_{6}(\mathrm{t})=\mathrm{Q}_{60}(\mathrm{t})[\mathrm{c}] \mathrm{H}_{0}(\mathrm{t})$

$\mathrm{H}_{7}(\mathrm{t})=\left(\mathrm{Q}_{72}(\mathrm{t})+\mathrm{Q}_{72}{ }^{(8)}(\mathrm{t})\right)[\mathrm{c}] \mathrm{H}_{2}(\mathrm{t})+\mathrm{Q}_{74}(\mathrm{t})[\mathrm{c}] \mathrm{H}_{4}(\mathrm{t})$

$\mathrm{H}_{9}(\mathrm{t})=\mathrm{Q}_{9,10}(\mathrm{t})[\mathrm{c}]\left[1+\mathrm{H}_{10}(\mathrm{t})\right], \quad \mathrm{H}_{10}(\mathrm{t})=\left(\mathrm{Q}_{10,2}(\mathrm{t})[\mathrm{c}]+\mathrm{Q}_{10,2}{ }^{(11)}(\mathrm{t})\right)[\mathrm{c}] \mathrm{H}_{2}(\mathrm{t})$

Taking Laplace Transform of eq. (51-58) and solving for $H_{0}^{8}(s)$

where

$$
H_{0}^{8}(s)=\mathrm{N}_{6}(\mathrm{~s}) / \mathrm{D}_{3}(\mathrm{~s})
$$

$\mathrm{N}_{6}(\mathrm{~s})=\left(1-Q_{22}{ }^{(3) *}(\mathrm{~s})\right)\left\{Q^{8}{ }_{01}(\mathrm{~s})\left(Q_{12}^{8}(\mathrm{~s})+Q^{8}{ }_{14}(\mathrm{~s})\right)+Q^{8}{ }_{09}(\mathrm{~s}) Q_{9,10}^{8}(\mathrm{~s})\right\}$

$\mathrm{D}_{3}(\mathrm{~s})=\left(1-Q_{22}{ }^{(3) *}(\mathrm{~s})\right)\left\{1-\left(Q^{8}{ }_{01}(\mathrm{~s}) Q^{8}{ }_{14}(\mathrm{~s})+Q^{8}{ }_{07}(\mathrm{~s}) Q^{8}{ }_{74}(\mathrm{~s})\right) Q_{46}(5) *(\mathrm{~s}) Q_{60}^{8}(\mathrm{~s})\right\}-Q^{8}{ }_{20}(\mathrm{~s})\left\{Q^{8}{ }_{01}(\mathrm{~s}) Q^{8}{ }_{12}(\mathrm{~s})+\right.$ $Q^{8}{ }_{07}(\mathrm{~s})\left(Q^{8}{ }_{72}(\mathrm{~s})\right)+Q^{8}{ }_{72}^{(8)}(\mathrm{s})+$

$$
\left.Q^{8}{ }_{09}(\mathrm{~s}) Q_{9,10}^{8}(\mathrm{~s})\left(Q^{8}{ }_{10,2}(\mathrm{~s})+\mathrm{Q}_{10,2}^{(11)^{*}}(\mathrm{~s})\right)\right\}
$$

In the long run, $\mathrm{H}_{0}=\frac{N_{\mathrm{B}}(\mathrm{O})}{D_{\mathrm{g}} \mathrm{T}(\mathrm{O})}$ 


\section{International Journal of Innovative Research in Science, Engineering and Technology}

(An ISO 3297: 2007 Certified Organization)

Vol. 3, Issue 10, October 2014

where $\mathrm{N}_{6}(0)=\mathrm{p}_{20}\left(\mathrm{p}_{01}+\mathrm{p}_{09}\right)$ and $\mathrm{D}_{3}^{\prime}(0)$ is already defined.

\section{THE EXPECTED NUMBER OF VISITS BY THE REPAIRMAN FOR REPAIRING THE SWITCH IN $(0, T]$}

$\mathrm{V}_{0}(\mathrm{t})=\mathrm{Q}_{01}(\mathrm{t})[\mathrm{c}] \mathrm{V}_{1}(\mathrm{t})+\mathrm{Q}_{07}(\mathrm{t})[\mathrm{c}] \mathrm{V}_{7}(\mathrm{t})+\mathrm{Q}_{09}(\mathrm{t})[\mathrm{c}] \mathrm{V}_{9}(\mathrm{t})$

$\mathrm{V}_{1}(\mathrm{t})=\mathrm{Q}_{12}(\mathrm{t})[\mathrm{c}] \mathrm{V}_{2}(\mathrm{t})+\mathrm{Q}_{14}(\mathrm{t})[\mathrm{c}] \mathrm{V}_{4}(\mathrm{t}), \mathrm{V}_{2}(\mathrm{t})=\mathrm{Q}_{20}(\mathrm{t})[\mathrm{c}] \mathrm{V}_{0}(\mathrm{t})+\mathrm{Q}_{22}{ }^{(3)}(\mathrm{t})[\mathrm{c}] \mathrm{V}_{2}(\mathrm{t})$

$\mathrm{V}_{4}(\mathrm{t})=\mathrm{Q}_{46}{ }^{(3)}(\mathrm{t})[\mathrm{c}] \mathrm{V}_{6}(\mathrm{t}), \mathrm{V}_{6}(\mathrm{t})=\mathrm{Q}_{60}(\mathrm{t})[\mathrm{c}] \mathrm{V}_{0}(\mathrm{t})$

$\mathrm{V}_{7}(\mathrm{t})=\left(\mathrm{Q}_{72}(\mathrm{t})\left[1+\mathrm{V}_{2}(\mathrm{t})\right]+\mathrm{Q}_{72}{ }^{(8)}(\mathrm{t})\right)[\mathrm{c}] \mathrm{V}_{2}(\mathrm{t})+\mathrm{Q}_{74}(\mathrm{t})[\mathrm{c}] \mathrm{V}_{4}(\mathrm{t})$

$\mathrm{V}_{9}(\mathrm{t})=\mathrm{Q}_{9,10}(\mathrm{t})[\mathrm{c}] \mathrm{V}_{10}(\mathrm{t}), \mathrm{V}_{10}(\mathrm{t})=\left(\mathrm{Q}_{10,2}(\mathrm{t})+\mathrm{Q}_{10,2}(11)(\mathrm{t})\right)[\mathrm{c}] \mathrm{V}_{2}(\mathrm{t})$

Taking Laplace-Stieltjes transform of eq. (61-68) and solving for $V_{0}^{*}(s)$

where

$V_{0}^{*}(s) \quad=\mathrm{N}_{7}(\mathrm{~s}) / \mathrm{D}_{4}(\mathrm{~s})$

$\mathrm{N}_{7}(\mathrm{~s})=Q^{8}{ }_{07}(\mathrm{~s}) Q^{8}{ }_{72}(\mathrm{~s})\left(1-Q_{22}{ }^{(3)^{*}}(\mathrm{~s})\right)$ and $\mathrm{D}_{4}(\mathrm{~s})$ is the same as $\mathrm{D}_{3}(\mathrm{~s})$

In the long run, $\mathrm{V}_{0}=\frac{N_{7}(0)}{D_{4}(0)}$

where

$\mathrm{N}_{7}(0)=\mathrm{p}_{20} \mathrm{p}_{07} \mathrm{p}_{72}$ and $\mathrm{D}^{\prime}{ }_{3}(0)$ is already defined.

\section{XIV.GAIN- FUNCTION ANALYSIS}

The gain- function of the system considering mean up-time, expected busy period of the system under voltage fluctuations when the units stops automatically, expected busy period of the server for repair of unit due to nonavailability of water resulting failure of hydroelectric power and expected busy period of the server to repair the switch, expected number of visits by the repairman for unit failure, expected number of visits by the repairman for switch failure.

The expected total cost-benefit incurred in $(0, t]$ is

$\mathrm{C}(\mathrm{t})=$ Expected total revenue in $(0, \mathrm{t}]$ - expected total repair cost for switch in $(0, \mathrm{t}]$

- expected total repair cost for repairing the units due to voltage fluctuations when the units automatically stop in $(0, t]$

- expected busy period of the system under non-availability of water resulting failure of hydroelectric power - expected number of visits by the repairman for repairing the switch in $(0, t]$

- expected number of visits by the repairman for repairing of the dissimilar units in $(0, t]$

The expected total cost per unit time in steady state is

$$
\begin{aligned}
\mathrm{C} & =\lim _{t \rightarrow \infty}(C(t) / t)=\lim _{s \rightarrow 0}\left(s^{2} C(s)\right) \\
& =\mathrm{K}_{1} \mathrm{~A}_{0}-\mathrm{K}_{2} \mathrm{P}_{0}-\mathrm{K}_{3} \mathrm{~B}_{0}-\mathrm{K}_{4} \mathrm{R}_{0}-\mathrm{K}_{5} \mathrm{~V}_{0}-\mathrm{K}_{6} \mathrm{H}_{0}
\end{aligned}
$$

Where

$\mathrm{K}_{1}$ - revenue per unit up-time,

$\mathrm{K}_{2}$ - cost per unit time for which the system is under switch repair,

$\mathrm{K}_{3}$ - cost per unit time for which the system is under repair due to voltage fluctuations when units automatically stop,

$\mathrm{K}_{4}$ - cost per unit time for which the system is under repair due to non-availability of water resulting failure of hydroelectric power,

$\mathrm{K}_{5}-$ cost per visit by the repairman for which switch repair,

$\mathrm{K}_{6}$ - cost per visit by the repairman for units repair. 


\section{IJIRSET \\ International Journal of Innovative Research in Science, Engineering and Technology}

ISSN: 2319-8753

(An ISO 3297: 2007 Certified Organization)

Vol. 3, Issue 10, October 2014

\section{XV.CONCLUSION}

After studying the system, we have analyzed graphically that when the failure rate due to voltage fluctuations, failure rate due to non-availability of water resulting failure of hydroelectric power increases, the MTSF and steady state availability decreases and the cost function decreased as the failure increases.

\section{REFERENCES}

[1] Barlow, R.E. and Proschan, F., Mathematical theory of Reliability, John Wiley, New York, 1965

[2] Gnedanke, B.V., Belyayar, Yu.K. and Soloyer, A.D., Mathematical Methods of Relability Theory, Academic Press, New York, 1969.

[3] Dhillon, B.S. and Natesen, J, Stochastic Anaysis of outdoor Power Systems in fluctuating environment, Microelectron. Reliab., 23, 867-881, 1983.

[4] Goel, L.R., Sharma,G.C. and Gupta, Rakesh Cost Analysis of a Two-Unit standby system with different weather conditions, Microelectron. Reliab. , 25, 665-659,1985.

[5] Goel,L.R. ,Sharma G.C. and Gupta Parveen , Stochastic Behavior and Profit Analysis of a redundant system with slow switching device, Microelectron Reliab., 26, 215-219., 1986.

[6]Cao, Jinhua, Stochastic Behavior of a Man Machine System Operating under Changing Environment Subject to a Markov Process with two States, Microelectron. Reliab., 28, pp. 373-378, 1989. 